PREPARED FOR SUBMISSION TO JCAP

\title{
The reconstruction of tachyon inflationary potentials
}

\section{Qin Fei, Yungui Gong, ${ }^{1}$ Jiong Lin, and Zhu Yi}

School of Physics, Huazhong University of Science and Technology,1037 LuoYu Rd, Wuhan, Hubei 430074, China

E-mail: feiqin@hust.edu.cn, yggong@mail.hust.edu.cn, 707751841@qq.com, yizhu92@hust.edu.cn

\begin{abstract}
We derive a lower bound on the field excursion for the tachyon inflation, which is determined by the amplitude of the scalar perturbation and the number of $e$-folds before the end of inflation. Using the relation between the observables like $n_{s}$ and $r$ with the slow-roll parameters, we reconstruct three classes of tachyon potentials. The model parameters are determined from the observations before the potentials are reconstructed, and the observations prefer the concave potential. We also discuss the constraints from the reheating phase preceding the radiation domination for the three classes of models by assuming the equation of state parameter $w_{r e}$ during reheating is a constant. Depending on the model parameters and the value of $w_{r e}$, the constraints on $N_{r e}$ and $T_{r e}$ are different. As $n_{s}$ increases, the allowed reheating epoch becomes longer for $w_{r e}=-1 / 3,0$ and $1 / 6$ while the allowed reheating epoch becomes shorter for $w_{r e}=2 / 3$.
\end{abstract}

ArXiv ePrint: 1705.02545

\footnotetext{
${ }^{1}$ Corresponding author
} 


\section{Contents}

1 Introduction $\quad 1$

2 Tachyon inflation $\quad 2$

2.1 slow-roll inflation 4

2.2 Perturbations 5

2.3 Field excursion $\quad 7$

2.4 Summary of the relations $\quad 7$

2.5 The relation to generalized $\beta$-function formalism 8

3 The reconstruction $\quad 8$

3.1 The constant slow-roll inflation 9

$\begin{array}{ll}3.2 \text { The power-law parametrization of } n_{s} & 12\end{array}$

$\begin{array}{lll}3.3 & \text { The power-law parametrization of } r & 13\end{array}$

4 Reheating $\quad 16$

5 Conclusions and Discussions $\quad 20$

\section{Introduction}

Inflation not only provides the solution to the monopole, horizon and flatness problems, but also provides the seeds for the large scale structure of the Universe. The inflationary phase is usually driven by the potential or vacuum energy of a scalar field called the inflaton with a flat potential. Motivated by string theory, the tachyon condensate with the effective Dirac-Born-Infeld action is an interesting scalar field and the cosmological consequences of the rolling tachyon were widely studied [1-5]. Tachyon inflation also provides the almost scale invariant power spectrum [6-8]. To compare inflationary models with the observations, we need to calculate the observables $n_{s}$ and $r$ for a pivotal scale $k_{*}$, and the results are usually expressed in terms of the number of $e$-folds $N_{*}$ before the end of inflation at the horizon exit of the pivotal scale. For example, the chaotic inflation with the power-law potential $\phi^{p}$ gives $n_{s}=1-(p+2) /\left(2 N_{*}\right)$ and $r=4 p / N_{*}[9]$, the Starobinsky model gives $n_{s}=1-2 / N_{*}$ and $r=12 / N_{*}^{2}[10]$ which is consistent with the Planck 2015 results $n_{s}=0.9645 \pm 0.0049$ and $r_{0.002}<$ 0.10 [11]. Therefore, we can parameterize the observables or the slow-roll parameters with $N$ for inflationary models $[12,13]$. Furthermore, by parameterizing the slow-roll parameters or the observable $n_{s}$ with $N$, we can constrain the model parameters easily and reconstruct the inflationary potentials [13-34]. For the ultra slow-roll inflation $[35,36]$, the slow-roll parameter $\eta=\ddot{\phi} /(H \dot{\phi})$ is a constant and the reconstruction was discussed in [37-40]. The reconstruction method was also applied to tachyon inflation by parameterizing the slow-roll parameter $\epsilon$ or equivalently $r$ with $N$ [41]. 
In addition to the constraints on $n_{s}$ and $r$, it was proposed that the reheating phase preceding the radiation domination may provide further constraints on inflationary models [42]. Assuming that the effective equation of state parameter $w_{r e}$ is a constant, we can relate the total number of $e$-folds during reheating with $N_{*}$ and the energy scale at the end of inflation [42-47]. In this paper, we use the reconstruction method by assuming either constant or simple inverse power-law parametrization to reconstruct tachyon potentials and discuss additional constraints from reheating.

The paper is organized as follows. In section II, we review the tachyon inflation and the reconstruction method. The lower bound on the field excursion are derived, and the relation between the reconstruction method and the generalized $\beta$-function method is also discussed. In section III, we reconstruct the classes of potentials for the constant $\eta_{V}$, the simple parametrization $n_{s}=1-p /(N+A)$ and the inverse power-law parametrization $r=16 \gamma /(N+\alpha)^{\beta}$. By assuming that the equation of state parameter $w_{r e}$ during reheating is a constant, we discuss the constraints on reheating for the three models in section IV, the paper is concluded in section V.

\section{Tachyon inflation}

For more general scalar fields, the kinetic term may not take the standard canonical form. In particular, the tachyon condensate in the string theory can be described by an effective scalar field with nonlinear kinetic term which drives inflation even without the help of the potential. The effective action for the rolling tachyon is

$$
S_{T}=-\int d^{4} x \sqrt{-g} V(T) \sqrt{1+g^{\mu \nu} \partial_{\mu} T \partial_{\nu} T}
$$

Applying the Arnowitt-Deser-Misner (ADM) metric [48],

$$
d s^{2}=-\mathscr{N}^{2} d t^{2}+h_{i j}\left(d x^{i}+N^{i} d t\right)\left(d x^{j}+N^{j} d t\right)
$$

the gravitational and tachyon action becomes

$$
S=\int d^{4} x \mathscr{N} \sqrt{h}\left\{\frac{1}{2}\left[{ }^{(3)} R+\frac{1}{\mathscr{N}^{2}}\left(E^{i j} E_{i j}-E^{2}\right)\right]-V\left(1-\frac{\dot{T}^{2}}{\mathscr{N}^{2}}\right)^{\frac{1}{2}}\right\}
$$

where $\mathscr{N}$ and $N^{i}$ are the lapse and the shift functions, respectively, all the spatial indices are raised and lowered by the metric $h_{i j}$ for the three dimensional space, $\dot{T}=$ $d T / d t$

$$
E_{i j}=\frac{1}{2}\left(\dot{h}_{i j}-\nabla_{i} N_{j}-\nabla_{j} N_{i}\right),
$$

$E=h^{i j} E_{i j}$, the extrinsic curvature $K_{i j}=E_{i j} / \mathscr{N}$, and the covariant derivative is with respect to the three dimensional spatial metric $h_{i j}$. Note that we take $M_{p l}=$ $1 / \sqrt{8 \pi G}=1$. Since the lapse and shift functions $\mathscr{N}$ and $N_{i}$ contain no time derivative, 
the variations with respect to them give the corresponding Hamiltonian and momentum constraints,

$$
\begin{gathered}
\nabla_{i}\left[\frac{1}{\mathscr{N}}\left(E_{j}^{i}-h_{i j} E\right)\right]=0 \\
{ }^{(3)} R-\frac{1}{\mathscr{N}^{2}}\left(E^{i j} E_{i j}-E^{2}\right)-2 V\left(1-\frac{\dot{T}^{2}}{\mathscr{N}^{2}}\right)^{-\frac{1}{2}}=0 .
\end{gathered}
$$

For the homogeneous and isotropic background, $\mathscr{N}=1, N_{i}=0$ and $h_{i j}=a^{2} \delta_{i j}$, the Hamiltonian constraint (2.6) becomes the Friedmann equation

$$
H^{2}=\frac{1}{3} \frac{V}{\sqrt{1-\dot{T}^{2}}},
$$

and the momentum constraint satisfies automatically. The energy density and the equation of state for the tachyon field are

$$
\begin{gathered}
\rho=\frac{V}{\sqrt{1-\dot{T}^{2}}}, \\
w=\frac{p}{\rho}=\dot{T}^{2}-1 .
\end{gathered}
$$

The equation of motion for the tachyon field is

$$
\frac{\ddot{T}}{1-\dot{T}^{2}}+3 H \dot{T}+\frac{V_{, T}}{V}=0,
$$

where $V_{, T}=d V / d T$. Combining eqs. (2.7) and (2.10), we get

$$
\dot{H}=-\frac{3}{2} H^{2} \dot{T}^{2}
$$

Before we review the slow-roll inflation and perturbations, we discuss the inflationary attractor [49] first. Combining eqs. (2.7) and (2.11), we get the Hamilton-Jacobi equation

$$
V^{2}=9 H^{4}-4(d H / d T)^{2} .
$$

Suppose $H_{0}(T)$ is a solution to eq. (2.12), either inflationary or noninflationary, then we consider a perturbation $\delta H(T)$ around $H_{0}(T)$, i.e., another trajectory $H_{0}(T)+\delta H(T)$ which satisfies eq. (2.12). To the linear perturbation, we get

$$
9 H_{0}^{3} \delta H=2\left(d H_{0} / d T\right)(d \delta H / d T) .
$$

In terms of the number of e-folds $N$ before the end of inflation, we get the solution

$$
\delta H(T)=\delta H\left(T_{i}\right) \exp \left[-3\left(N_{i}-N\right)\right] .
$$

If $H_{0}(T)$ is an inflationary solution, then all linear perturbations approach it exponentially as the tachyon rolls down, so inflationary attractor exists if the potential is able to support inflation. 


\section{1 slow-roll inflation}

From eq. (2.11), we get

$$
\frac{\ddot{a}}{a}=\dot{H}+H^{2}=H^{2}\left(1-\frac{3}{2} \dot{T}^{2}\right)
$$

The condition for inflation $\ddot{a}>0$ requires $\dot{T}^{2}<2 / 3$. If the tachyon field satisfies the slow-roll conditions

$$
\begin{aligned}
& \dot{T}^{2} \ll 1, \\
& \ddot{T} \ll 3 H \dot{T},
\end{aligned}
$$

then the background equations during inflation are

$$
\begin{gathered}
H^{2} \approx \frac{V}{3}, \\
3 H \dot{T} \approx-V_{, T} / V .
\end{gathered}
$$

By using the number of $e$-folds $N(t)=\ln \left(a_{e} / a\right)$ before the end of inflation,

$$
N(t)=\int_{t}^{t_{e}} H(t) d t
$$

we introduce the horizon-flow slow-roll parameters [50]

$$
\begin{gathered}
\epsilon_{0}=\frac{H_{*}}{H}, \\
\epsilon_{i+1}=-\frac{d \ln \left|\epsilon_{i}\right|}{d N},
\end{gathered}
$$

where the subscript $e$ denotes the end of inflation, the subscript $*$ denotes the horizon crossing and we choose $H_{*}$ as the Hubble parameter at the horizon crossing for a particular scale, for example, $k_{*}=0.002 \mathrm{Mpc}^{-1}$. For the tachyon field, the first two slow-roll parameters are [8]

$$
\begin{gathered}
\epsilon=\epsilon_{1}=-\frac{\dot{H}}{H^{2}}=\frac{3}{2} \dot{T}^{2} \approx \frac{1}{2} \frac{V_{, T}^{2}}{V^{3}}, \\
\eta=\epsilon_{2}=2 \frac{\ddot{T}}{H \dot{T}} \approx-2 \frac{V_{, T T}}{V^{2}}+3 \frac{V_{, T}^{2}}{V^{3}} .
\end{gathered}
$$

From eqs. (2.20) and (2.23), we get

$$
N(T)= \pm \sqrt{\frac{3}{2}} \int_{T}^{T_{e}} \frac{H}{\sqrt{\epsilon}} d T \approx \int_{T_{e}}^{T} \frac{V^{2}}{V_{, T}} d T,
$$

where the \pm sign is the same as the sign of $\dot{T}$. 


\subsection{Perturbations}

For convenience, we choose the flat gauge,

$$
\begin{aligned}
& \delta T(x, t)=0, \mathscr{N}=1+N_{1}, N_{i}=\partial_{i} \psi+N_{i}^{T}, \\
& h_{i j}=a^{2}\left(\left(1+2 \zeta+2 \zeta^{2}\right) \delta_{i j}+\gamma_{i j}+\frac{1}{2} \gamma_{i l} \gamma_{l j}\right),
\end{aligned}
$$

where $\partial^{i} N_{i}^{T}=0, \zeta$ and $\gamma_{i j}$ denote the scalar and tensor fluctuations respectively, the tensor perturbation satisfies $\partial_{i} \gamma^{i j}=0$ and $h^{i j} \gamma_{i j}=0$. Note that $N_{1}, \psi, N_{i}^{T}, \zeta$ and $\gamma_{i j}$ are first order quantities. Substituting eq. (2.26) into the momentum constraint (2.5) and the Hamiltonian constraint (2.6), to the first order, we get the solution $N_{i}^{T}=0$ and

$$
\begin{aligned}
N_{1} & =\frac{\dot{\zeta}}{H} \\
\psi & =-\frac{\zeta}{a^{2} H}+\chi, \\
\nabla^{2} \chi & =\frac{3}{2} \frac{\dot{T}^{2}}{1-\dot{T}^{2}} \dot{\zeta} .
\end{aligned}
$$

Combining the solution (2.27) with the background equations (2.7), (2.10) and (2.11), to the second order of perturbation, the action (2.3) for the scalar perturbation becomes

$$
S=-\frac{3}{2} \int d^{4} x\left[a \dot{T}^{2}\left(\partial_{i} \zeta\right)^{2}-a^{3} \frac{\dot{T}^{2}}{1-\dot{T}^{2}} \dot{\zeta}^{2}\right]
$$

Using the canonically normalized field $v=z \zeta$, where

$$
z=\frac{\sqrt{3} a \dot{T}}{\sqrt{1-\dot{T}^{2}}}
$$

the action (2.28) becomes

$$
S=\int d^{3} x d \tau \frac{1}{2}\left[v^{\prime 2}-c_{s}^{2}\left(\partial_{i} v\right)^{2}+\frac{z^{\prime \prime}}{z} v^{2}\right],
$$

where the prime denotes the derivative with respect to the conformal time $\tau=\int d t / a$, and the effective sound speed is $c_{s}^{2}=1-\dot{T}^{2}[6]$. In terms of the slow-roll parameters, we get [8]

$$
\frac{z^{\prime \prime}}{z} \approx a^{2} H^{2}\left(2-\epsilon+\frac{3}{2} \eta\right)
$$

To discuss the quantum fluctuations, we define the operator

$$
\hat{v}(\tau, \vec{x})=\int \frac{d^{3} k}{(2 \pi)^{3}}\left[v_{k}(\tau) \hat{a}_{k} e^{i \vec{k} \cdot \vec{x}}+v_{k}^{*}(\tau) \hat{a}_{k}^{\dagger} e^{-i \vec{k} \cdot \vec{x}}\right],
$$


where the creation and annihilation operators satisfy the standard commutation relations

$$
\begin{aligned}
& {\left[\hat{a}_{k}, \hat{a}_{k^{\prime}}^{\dagger}\right]=(2 \pi)^{3} \delta^{3}\left(\vec{k}-\overrightarrow{k^{\prime}}\right),}
\end{aligned}
$$

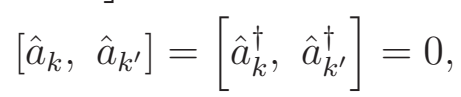

and the mode functions obey the normalization condition

$$
v_{k}^{\prime} v_{k}^{*}-v_{k} v_{k}^{* \prime}=-i .
$$

We choose the Bunch-Davis vacuum defined by $\hat{a}_{k}|0\rangle=0$. Varying the action (2.30) and using eq. (2.31), we obtain the Mukhanov-Sasaki equation for the mode function $v_{k}(\tau)[8]$

$$
v_{k}^{\prime \prime}+\left(c_{s}^{2} k^{2}-\frac{\nu^{2}-1 / 4}{\tau^{2}}\right) v_{k}=0
$$

where

$$
\nu=\frac{3}{2}+\epsilon+\frac{1}{2} \eta
$$

Solving eq. (2.35) with the condition (2.34), we find that outside the horizon, the scalar perturbation is almost a constant,

$$
\left|\zeta_{k}\right|=\frac{\left|v_{k}\right|}{z}=2^{\nu-\frac{5}{2}} \frac{\Gamma(\nu)}{\Gamma(3 / 2)} \frac{H(1-\epsilon)^{\nu-1 / 2}}{c_{s}^{1 / 2} k^{3 / 2} \epsilon^{1 / 2}}\left(\frac{c_{s} k}{a H}\right)^{\frac{3}{2}-\nu} .
$$

Therefore, the power spectrum of the scalar perturbation is [8]

$$
P_{\zeta}=\frac{k^{3}}{2 \pi^{2}}\left|\zeta_{k}\right|^{2}=\left.\left[1-\left(\frac{5}{3}+2 C\right) \epsilon-C \eta\right] \frac{H^{2}}{8 \pi^{2} \epsilon}\right|_{c_{s} k=a H}
$$

where $C=\gamma+\ln 2-2 \approx-0.72$. The amplitude of the scalar perturbation is

$$
A_{s}=\left.\frac{H^{2}}{8 \pi^{2} \epsilon}\right|_{c_{s} k=a H}
$$

The scalar spectral tilt is $[6,8]$

$$
n_{s}-1=\left.\frac{d \ln P_{\zeta}}{d \ln k}\right|_{c_{s} k=a H}=-2 \epsilon-\eta .
$$

For the tensor perturbation, to the second order, the action (2.3) becomes

$$
S=\frac{1}{8} \int d^{4} x\left[a^{3}\left(\dot{\gamma}_{i j}\right)^{2}-a\left(\gamma_{i j, k}\right)^{2}\right],
$$

so the tensor spectrum is [8]

$$
P_{T}=\left.[1-2(1+C) \epsilon] \frac{2 H^{2}}{\pi^{2}}\right|_{k=a H} .
$$


The tensor spectral tilt is [8]

$$
n_{T}=-2 \epsilon[1+\epsilon+(1+C) \eta] .
$$

The tensor to scalar ratio is $[6,8]$

$$
r=16 \epsilon\left[1-\frac{1}{3} \epsilon+C \eta\right] .
$$

\section{$2.3 \quad$ Field excursion}

If $\epsilon$ is a monotonic function and $H$ decreases during inflation, then from eqs. (2.25) and (2.39), we get

$$
N_{*} \leq \sqrt{\frac{3}{2}} \frac{H_{*}}{\sqrt{\epsilon\left(T_{*}\right)}}\left|T_{e}-T_{*}\right|=\sqrt{12 \pi^{2} A_{s}} M_{p l} \Delta T .
$$

In the last equality, we write out $M_{p l}$ explicitly. Therefore, similar to the Lyth bound $[51,52]$, there is a lower bound on the field excursion for the tachyon,

$$
M_{p l} \Delta T \geq \frac{N_{*}}{\sqrt{12 \pi^{2} A_{s}}} \approx 1.18 \times 10^{5}\left(\frac{N_{*}}{60}\right),
$$

where we use the observational value $\ln \left(10^{10} A_{s}\right)=3.094$ [11].

\subsection{Summary of the relations}

From eqs. (2.20) and (2.23), we get

$$
\epsilon=\frac{3}{2} \dot{T}^{2} \approx \frac{V_{, N}}{2 V}
$$

From eq. (2.24), we get

$$
\eta=\frac{2 \ddot{T}}{H \dot{T}}=-\frac{d \ln \epsilon}{d N} .
$$

Substituting eqs. (2.47) and (2.48) into eq. (2.40), we get

$$
n_{s}-1=-2 \epsilon+\frac{d \ln \epsilon}{d N}=\left(\ln \frac{V_{, N}}{V^{2}}\right)_{, N} .
$$

From these relations, we see that once we parameterize one of the observable $n_{s}$ and $r$ or the slow-roll parameters $\epsilon$ and $\eta$ by $N$, we can derive all other parameters and the potential $V(N)$. Additionally, if we use the following relation

$$
d T \approx \pm \frac{\sqrt{V_{, N}}}{V} d N
$$

to derive the function $T(N)$, then we can reconstruct the potential $V(T)$, where the \pm sign is the same as the sign of $d V / d T$. Alternatively, we can derive $V(T)$ with the following relation,

$$
\frac{d V}{d T}=\frac{d V}{d N} \frac{d N}{d T}= \pm V^{3 / 2}(2 \epsilon)^{1 / 2}
$$


Therefore, by specifying one of the functions $\epsilon(N), \eta(N), n_{s}(N), T(N)$ and $V(N)$, we can derive the observable $n_{s}(N)$ and $r(N)$ and reconstruct the potential $V(T)$ within the observable scales. Since we approximate the power spectrum to the first order of the slow-roll parameters by assuming that the higher order corrections are small, the reconstruction is valid only under the slow-roll approximation and the reconstructed potential satisfies the slow-roll condition. Outside the slow-roll regime, the potential can be rather different.

\subsection{The relation to generalized $\beta$-function formalism}

In the generalized $\beta$-function formalism [53-55], the superpotential $W(T)=-2 H(T)$ and the $\beta$-function is defined as

$$
\beta(T)=-2\left(-p_{, X}\right)^{-1 / 2} \frac{W_{, T}}{W},
$$

where $-p_{, X}=\rho=V(T) / \sqrt{1-\dot{T}^{2}}$. By using the Friedman eqs. (2.7) and (2.10), it can be shown that $\beta^{2}(T)=3 \dot{T}^{2}$, so

$$
\beta(T)=-\sqrt{2 \epsilon} \approx-\frac{V_{, T}}{V^{3 / 2}} .
$$

For a given $\beta$-function, we can reconstruct the potential $V(T)$ from the above relation. Once the potential is reconstructed, we can derive the parametrization from eq. (2.53). Alternatively, if we parameterize the slow-roll parameters or the observables by $N$, we can reconstruct the potential and derive the $\beta$-function from eq. (2.53).

\section{The reconstruction}

The simplest parametrization is the constant parametrization. Let us consider $\epsilon=r / 16$ being a constant first. For this case, we get $\eta=0$ from eq. (2.48), and $n_{s}=1-2 \epsilon=$ $1-r / 8$ from eq. (2.49). The result $r=8\left(1-n_{s}\right)$ is excluded by the Planck 2015 observations at the $3 \sigma$ level. If we assume that $\eta$ is a constant, then from eq. (2.48), we get

$$
\epsilon=e^{-\eta N},
$$

where we choose the integration constant so that $\epsilon(N=0)=1$. Plugging the result (3.1) into eq. (2.49), we get

$$
n_{s}=1-\frac{r}{8}+\frac{1}{N} \ln \left(\frac{r}{16}\right) .
$$

The result is also excluded by the Planck 2015 observations at the $3 \sigma$ level. Now let us consider the case that $n_{s}$ is a constant. From eq. (2.49), we get

$$
\begin{gathered}
\epsilon=-\frac{\alpha e^{\alpha N}}{2 e^{\alpha N}+\alpha D} \\
V=-V_{0}\left[1+\frac{2}{\alpha D} e^{\alpha N}\right]^{-1},
\end{gathered}
$$


where the constant $\alpha=n_{s}-1$. The end of inflation $\epsilon(N=0)=1$ gives $D=-(2+\alpha) / \alpha$. The tensor to scalar ratio is

$$
r=\frac{16\left(1-n_{s}\right)}{2-\left(1+n_{s}\right) e^{\left(1-n_{s}\right) N}} .
$$

This result is again excluded by the Planck 2015 observations at the $3 \sigma$ level.

\subsection{The constant slow-roll inflation}

Now Let us consider the slow-roll parametrization with constant $\eta_{H}$,

$$
\eta_{H}=-\frac{\ddot{H}}{2 H \dot{H}}=\epsilon+\frac{1}{2} \frac{d \ln \epsilon}{d N},
$$

where the constant $\left|\eta_{H}\right|<1$. By imposing the condition $\epsilon(N=0)=1$, the solution to eq. (3.6) is

$$
\epsilon=\frac{r}{16}=\frac{\eta_{H}}{1+\left(\eta_{H}-1\right) \exp \left(-2 \eta_{H} N\right)}
$$

Substituting the result (3.7) into eqs. (2.49) and (2.50), we get

$$
n_{s}-1=2 \eta_{H}-\frac{4 \eta_{H}}{1+\left(\eta_{H}-1\right) \exp \left(-2 \eta_{H} N\right)},
$$

and the reconstructed potential

$$
V=2 V_{0} \sec ^{2}\left[\sqrt{\eta_{H} V_{0}}\left(T-T_{0}\right)\right] .
$$

Comparing the results (3.7) and (3.8) with the Planck 2015 observations [11], we get the constraints on $\eta_{H}$ and $N_{*}$ and the results are shown in figure 1 . We see that the constant $\eta_{H}$ is not consistent with the observations at the $1 \sigma$ level if $N_{*} \leq 60$.

Next we consider the constant slow-roll parametrization

$$
\eta_{V}=2 \frac{V_{, T T}}{V^{2}} \approx \frac{d \ln \epsilon}{d N}+6 \epsilon,
$$

where the constant $\left|\eta_{V}\right|<1$. From the definition of $\eta_{V}$, we find that the potential $V(T)$ takes the form of the Weierstrass function. By imposing the condition $\epsilon(N=0)=1$, the solution to eq. (3.10) is

$$
\epsilon=\frac{r}{16}=-\frac{\eta_{V}}{\left(6-\eta_{V}\right) e^{-\eta_{V} N}-6} .
$$

Substituting eq. (3.11) into eq. (2.49), we get

$$
n_{s}-1=\frac{8 \eta_{V}}{\left(6-\eta_{V}\right) e^{-\eta_{V} N}-6}+\eta_{V}
$$

From eqs. (3.11) and (3.12), we get $\eta_{V}=n_{s}-1+r / 2$. Comparing the results (3.11) and (3.12) with the Planck 2015 observations [11], we get the constraints on $\eta_{V}$ and $N_{*}$ 

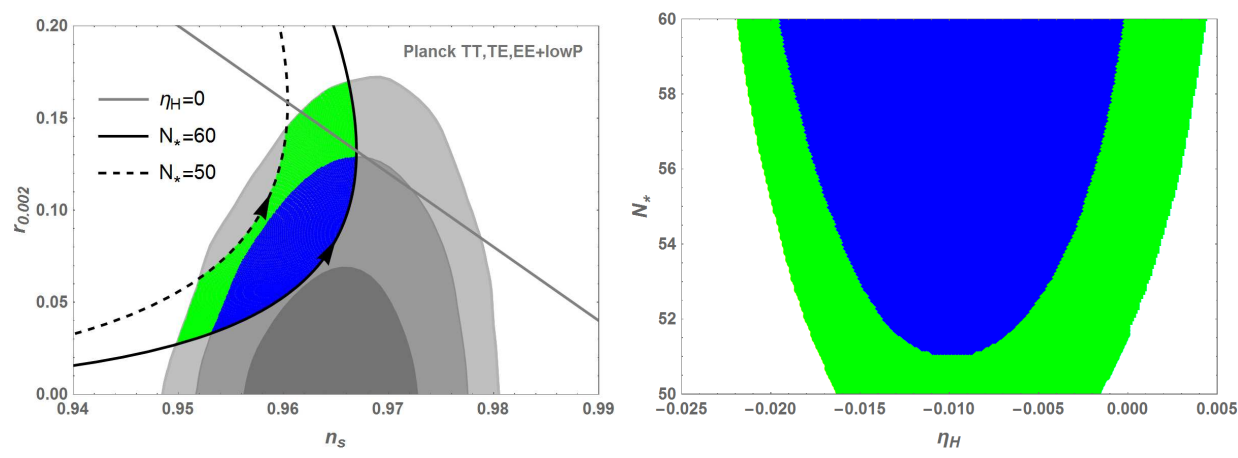

Figure 1. The marginalized $68 \%, 95 \%$ and $99.8 \%$ confidence level contours for $n_{s}$ and $r_{0.002}$ from Planck 2015 data [11] and the observational constraint on $\eta_{H}$. The left panel shows the $n_{s}-r$ contours and $\eta_{H}$ increases along the arrow direction. The right panel shows the $95 \%$ and $99.8 \%$ confidence level constraints on $\eta_{H}$ and $N_{*}$ and they are colored by the blue and green, respectively.

and the results are shown in figure 2 . For $N_{*}=60$, we get $-0.0374<\eta_{V}<-0.0142$ at the $1 \sigma$ level, $-0.0435<\eta_{V}<-0.0031$ at the $2 \sigma$ level and $-0.0473<\eta_{V}<0.0067$ at the $3 \sigma$ level. From figure 2, we see that $\eta_{V}<0$ is favored at more than $2 \sigma$ confidence level, so the concave potential is preferred. From now on, we call constant $\eta_{V}$ as the constant slow-roll inflation.

Substituting eq. (3.11) into eq. (2.47), we get

$$
V(N)=V_{0}\left|6 e^{\eta_{V} N}-6+\eta_{V}\right|^{\frac{1}{3}},
$$

where

$$
V_{0}=\frac{3 \pi^{2} A_{s} r}{2}\left|\frac{8-8 n_{s}-r}{\left(8-8 n_{s}-4 r\right)\left(7-n_{s}-r / 2\right)}\right|^{1 / 3} .
$$

From eq. (2.50), we get

$$
d T=\frac{2}{\eta_{V}} \sqrt{\frac{2\left|\eta_{V}\right|}{V_{0}}} \frac{1}{\left(6 x^{2}-6+\eta_{V}\right)^{2 / 3}} d x
$$

where $x=e^{\eta_{V} N / 2}$. The solution gives the Hypergeometric function

$$
T-T_{0}=\frac{2}{\eta_{V}} \sqrt{\frac{2\left|\eta_{V}\right|}{V_{0}}} \frac{\exp \left(\eta_{V} N / 2\right)}{\left(6-\eta_{V}\right)^{2 / 3}}{ }_{2} F_{1}\left(\frac{1}{2}, \frac{2}{3} ; \frac{3}{2} ; \frac{6 e^{\eta_{V} N}}{6-\eta_{V}}\right) \text {. }
$$

Combining eqs. (3.13) and (3.16), we can obtain the potential $V(T)$. If we take $\eta_{V}=-0.021$ and $N_{*}=60$, we get $n_{s}=0.968, r=0.022$ and $\Delta T=T_{*}-T_{e}=3.66 \times 10^{5}$, so the field excursion satisfies the bound (2.46). By using these parameters, we plot the potential in figure 3, and the slow-roll attractor for the potential is shown in figure 4 . 

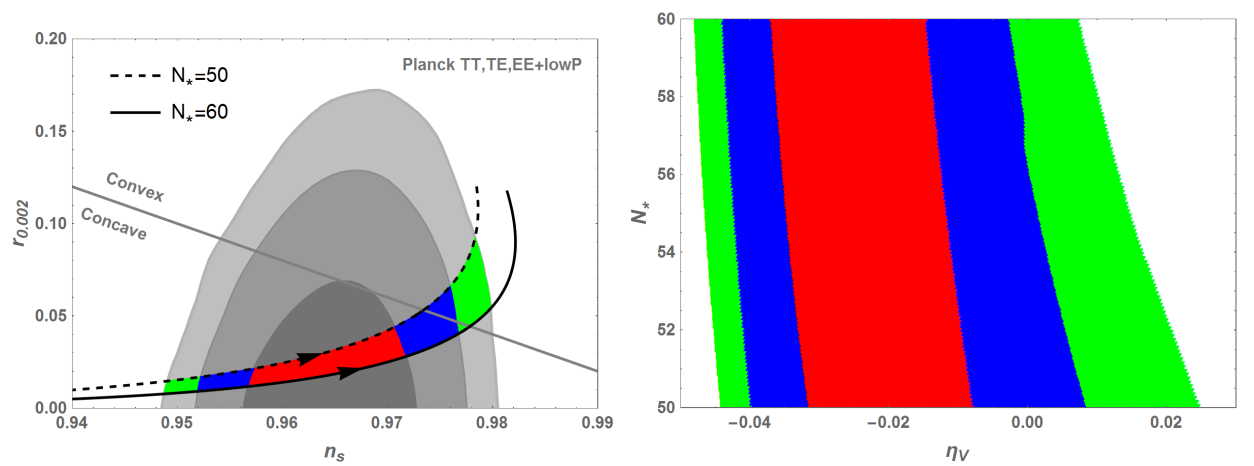

Figure 2. The marginalized $68 \%, 95 \%$ and $99.8 \%$ confidence level contours for $n_{s}$ and $r_{0.002}$ from Planck 2015 data [11] and the observational constraint on $\eta_{V}$. The left panel shows the $n_{s}-r$ contours and $\eta_{V}$ increases along the arrow direction. The right panel shows the $68 \%$, $95 \%$ and $99.8 \%$ confidence level constraints on $\eta_{V}$ and $N_{*}$ and they are colored by the red, blue and green, respectively.

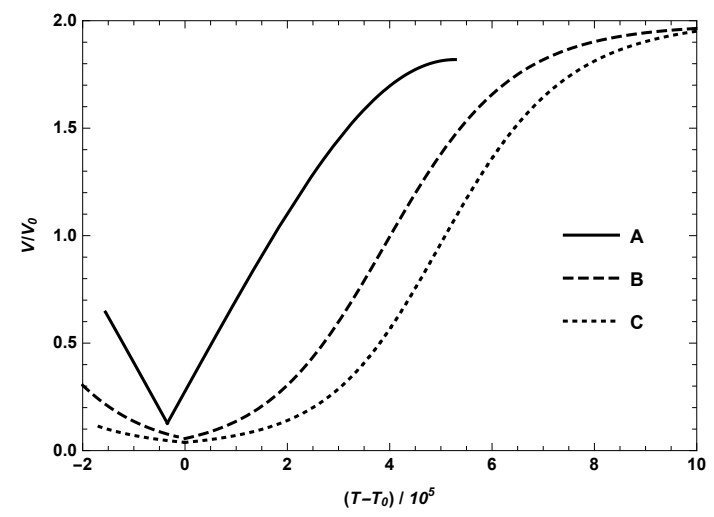

Figure 3. The reconstructed potentials normalized by $V_{0}$ from eq. (3.14). The solid line A corresponds to the potential for the constant slow-roll inflation, the dashed line B denotes the potential (3.25), and the dotted line $\mathrm{C}$ denotes the potential from eq. (3.45).

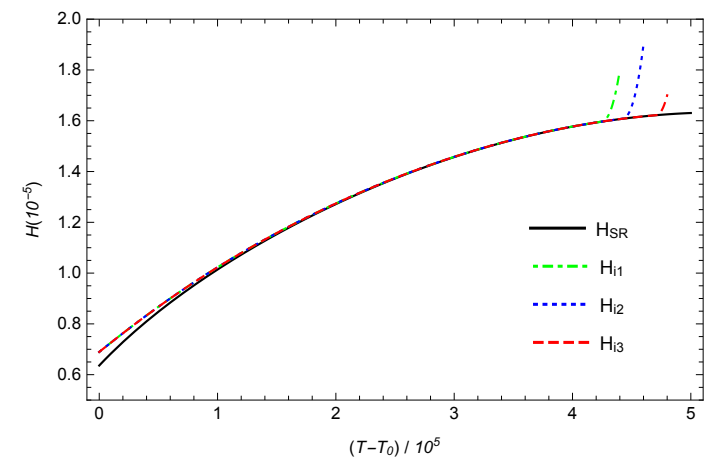

Figure 4. The solutions to the Hamilton-Jacobi equation (2.12) for the potential (3.13) with different initial values $H\left(T_{i}\right)$. The solid line labelled as $H_{\mathrm{SR}}$ corresponds to the slow-roll attractor. 


\subsection{The power-law parametrization of $n_{s}$}

The observational data favors $n_{s}=1-2 / N *$ with $N_{*}=60$, so we choose the parametrization

$$
n_{s}=1-\frac{p}{N+A}
$$

From eq. (2.49), we get

$$
\epsilon(N)= \begin{cases}\frac{p-1}{2(N+A)+2 C(p-1)(N+A)^{p}}, & p \neq 1, \\ \frac{1}{2(N+A)[C-\ln (N+A)]}, & p=1,\end{cases}
$$

and

$$
V(N)= \begin{cases}\frac{\tilde{V}_{0}(p-1)}{(p-1) C+(N+A)^{1-p}}, & p \neq 1, \\ \frac{\tilde{V}_{0}}{C-\ln (N+A)}, & p=1,\end{cases}
$$

where $C$ and $\tilde{V}_{0}$ are integration constants.

For convenience, let us consider the case $p=1$ first. At the end of inflation, $N=0$ and $\epsilon(N)=1$, so $C=(2 A)^{-1}+\ln A$, and we get

$$
\begin{gathered}
n_{s}=1-\frac{1}{N+A}, \\
r=\frac{16 A}{(N+A)\left(1-A \ln [(N+A) / A]^{2}\right)} .
\end{gathered}
$$

It is easy to show that the results are excluded by the Planck 2015 observations [11] at the $3 \sigma$ level.

For $p \neq 1$, from the condition $\epsilon(N=0)=1$, we get $p-1-2 A=2 C(p-1) A^{p}$, so the tensor to scalar ratio is

$$
r=\frac{16(p-1)}{2(N+A)+(p-1-2 A)(N+A)^{p} / A^{p}} .
$$

Note that if $C=0$, then $p=1+2 A$ and $r=8\left[N\left(1-n_{s}\right)-1\right] /(N-1 / 2)$. If $p>1+2 A$ with $A>0$, then $r \sim 1 / N^{p}$. In particular, for the case $p=2$, we get the familiar $\alpha$ attractor $n_{s}=1-2 / N_{*}$ and $r=12 \alpha / N_{*}^{2}$ for large $N$ and $C \neq 0$. Comparing the results (3.17) and (3.22) with the Planck 2015 observations [11], taking $N_{*}=60$, we get the constraints on $p$ and $A$ and the results are shown in figure 5. From figure 5, we see that the results are similar to those for canonical scalar field [25].

Now we proceed to derive the class of potentials. From eq. (2.50), we get

$$
d T=\tilde{V}_{0}^{-1 / 2}(N+A)^{-\frac{p}{2}} d N .
$$

For $p \neq 1$ and $p \neq 2$, we have

$$
N(T)+A=\left[\frac{(2-p) \sqrt{\tilde{V}_{0}}}{2}\left(T-T_{0}\right)\right]^{\frac{2}{2-p}} .
$$


Combining eqs. (3.19) and (3.24), for $p \neq 1+2 A, p \neq 1$ and $p \neq 2$, we get the inverse power-law potential $[8,56,57]$

$$
V(T)=V_{0}\left[1+\beta_{1}\left(T-T_{0}\right)^{\frac{2 p-2}{p-2}}\right]^{-1},
$$

where

$$
\begin{gathered}
V_{0}=\frac{2 \tilde{V}_{0}(p-1) A^{p}}{p-1-2 A}, \\
\beta_{1}=\frac{2 A^{p} \tilde{V}_{0}^{(p-1) /(p-2)}}{p-1-2 A}\left(\frac{2-p}{2}\right)^{2(p-1) /(p-2)},
\end{gathered}
$$

and

$$
V(T)=(p-1) \tilde{V}_{0}\left[\frac{(2-p) \sqrt{\tilde{V}_{0}}}{2}\left(T-T_{0}\right)\right]^{2(p-1) /(2-p)},
$$

for $p=1+2 A, p \neq 1$ and $p \neq 2$. If we take $p=1.934, A=0.446$ and $N_{*}=60$, we get $n_{s}=0.968, r=0.022$, and $\Delta T=T_{*}-T_{e}=5.4 \times 10^{5}$, so the bound $(2.46)$ is satisfied. With these model parameters, we plot the potential (3.25) in figure 3 , and the slow-roll attractor is shown in figure 6 .

For $p=2$, we have

$$
N(T)+A=\exp \left[\sqrt{\tilde{V}_{0}}\left(T-T_{0}\right)\right] .
$$

Combining eqs. (3.19) and (3.29), we get the potential for the $\alpha$ attractor (3.17) with $p=2$

$$
V(T)=V_{0}\left[1+\beta_{2} \exp \left(-\sqrt{\tilde{V}_{0}}\left(T-T_{0}\right)\right)\right]^{-1},
$$

where $A \neq 1 / 2$ and

$$
\begin{aligned}
V_{0} & =\frac{2 A^{2} \tilde{V}_{0}}{1-2 A}, \\
\beta_{2} & =\frac{2 A^{2}}{1-2 A},
\end{aligned}
$$

and

$$
V(T)=\tilde{V}_{0} \exp \left[\sqrt{\tilde{V}_{0}}\left(T-T_{0}\right)\right]
$$

for $A=1 / 2$ and $p=2$.

\subsection{The power-law parametrization of $r$}

In this subsection, we consider the parametrization

$$
\epsilon=\frac{r}{16}=\frac{\gamma}{(N+\alpha)^{\beta}},
$$



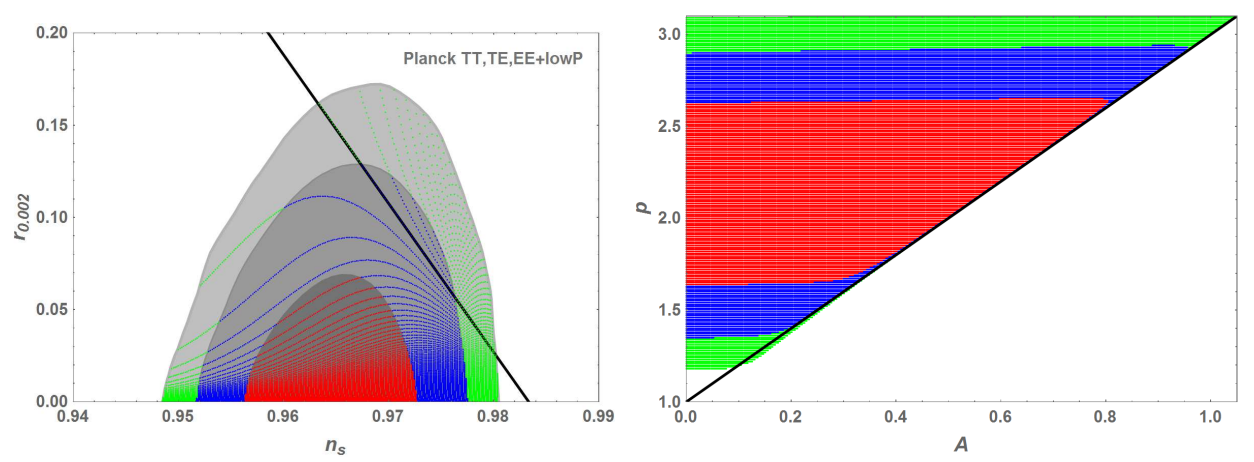

Figure 5. The marginalized $68 \%, 95 \%$ and $99.8 \%$ confidence level contours for $n_{s}$ and $r_{0.002}$ from Planck 2015 data [11] and the theoretical predictions for the parametrization (3.17) with $N_{*}=60$. The left panel shows the $n_{s}-r$ contours and the right panel shows the constraints on $p$ and $A$ for $N_{*}=60$. The red, blue and green regions correspond to $68 \%, 95 \%$ and $99.8 \%$ confidence levels, respectively. The solid black line denotes $p=1+2 A$.

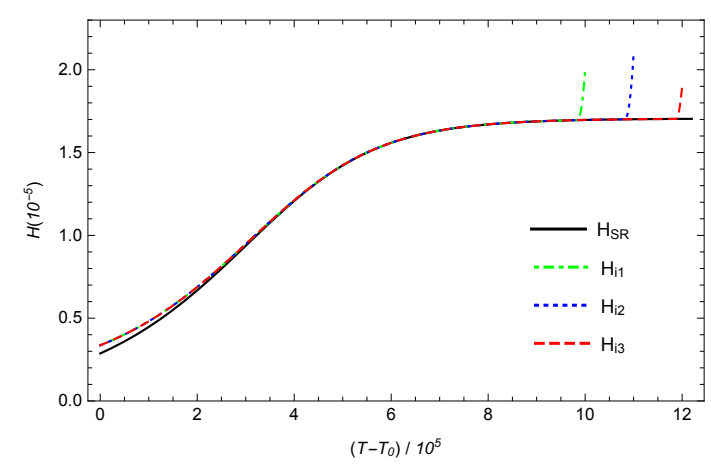

Figure 6. The solutions to the Hamilton-Jacobi equation (2.12) for the potential (3.25) with different initial values $H\left(T_{i}\right)$. The solid line labelled as $H_{\mathrm{SR}}$ corresponds to the slow-roll attractor.

where $\gamma=\alpha^{\beta}$ so that $\epsilon(N=0)=1$. Substituting the parametrization (3.34) into eq. (2.49), we get

$$
n_{s}-1=-2\left(\frac{\alpha}{N+\alpha}\right)^{\beta}-\frac{\beta}{N+\alpha} .
$$

Comparing the results (3.34) and (3.35) with the Planck 2015 observations [11], we get the constraints on $\alpha$ and $\beta$ and the results are shown in figure 7. Substituting eq. (3.34) into eq. (2.47), we get

$$
V(N)=\tilde{V}_{0}(N+\alpha)^{2 \alpha}
$$

for $\beta=1$, and

$$
V=\tilde{V}_{0} \exp \left[\frac{2 \gamma}{1-\beta}(N+\alpha)^{1-\beta}\right]
$$

for $\beta \neq 1$. So for $\beta \neq 1$, combining eqs. (3.34) and (3.37), we get

$$
\epsilon=\gamma\left[\frac{1-\beta}{2 \gamma} \ln \left(\frac{V}{\tilde{V}_{0}}\right)\right]^{-\frac{\beta}{1-\beta}} .
$$



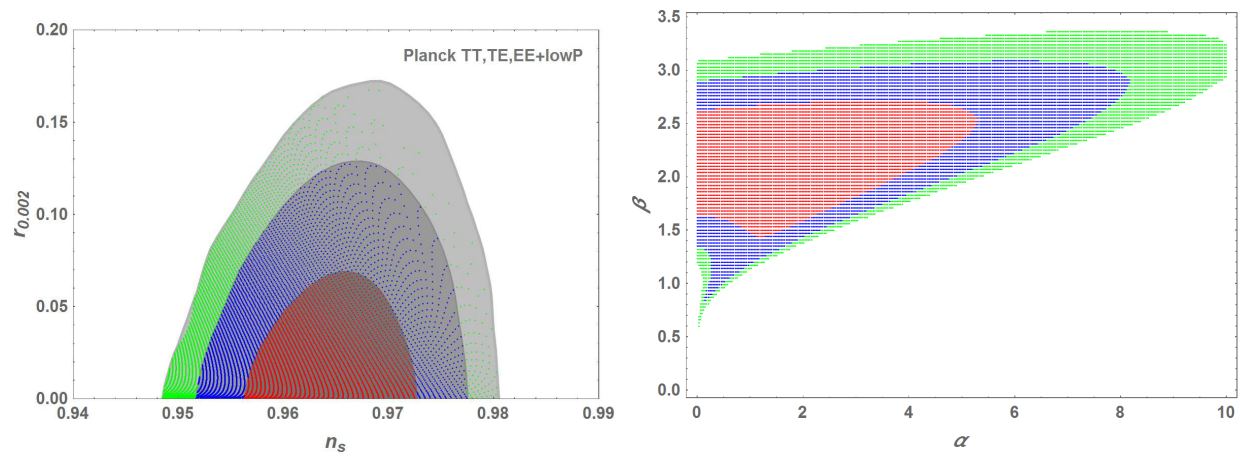

Figure 7. Same as figure 5 but for the parametrization (3.34). The left panel shows the $n_{s}-r$ contours and the right panel shows the constraints on $\alpha$ and $\beta$ for $N_{*}=60$. The red, blue and green regions correspond to $68 \%, 95 \%$ and $99.8 \%$ confidence levels, respectively.

Let us consider the case $\beta=1$ first. Substituting eq. (3.36) into eq. (2.50), we get

$$
\frac{d T}{d N}=\sqrt{\frac{2 \alpha}{\tilde{V}_{0}}}(N+\alpha)^{-\alpha-\frac{1}{2}}
$$

If $\alpha=1 / 2$, then

$$
N+\alpha=\exp \left[\sqrt{\tilde{V}_{0}}\left(T-T_{0}\right)\right] .
$$

Combining eq. (3.36) and eq. (3.40), we get the exponential potential [41] for $\beta=1$ and $\alpha=1 / 2$

$$
V=\tilde{V}_{0} \exp \left[\sqrt{\tilde{V}_{0}}\left(T-T_{0}\right)\right]
$$

If $\alpha \neq 1 / 2$, then

$$
N+\alpha=\left[\sqrt{\frac{\tilde{V}_{0}}{2 \alpha}}\left(\frac{1}{2}-\alpha\right)\left(T-T_{0}\right)\right]^{\frac{2}{1-2 \alpha}} .
$$

Combining eq. (3.36) and eq. (3.42), we get the power-law potential [41] for $\beta=1$ and $\alpha \neq 1 / 2$

$$
V=V_{0}\left(T-T_{0}\right)^{4 \alpha /(1-2 \alpha)},
$$

where

$$
V_{0}=\tilde{V}_{0}\left[\sqrt{\frac{\tilde{V}_{0}}{2 \alpha}}\left(\frac{1}{2}-\alpha\right)\right]^{\frac{4 \alpha}{1-2 \alpha}} .
$$

Now let us discuss the general case $\beta \neq 1$. Substituting eq. (3.38) into eq. (2.51), we get

$$
\sqrt{2 \gamma} d T= \pm\left[\frac{1-\beta}{2 \gamma} \ln \left(\frac{V}{\tilde{V}_{0}}\right)\right]^{\frac{\beta}{2(1-\beta)}} \frac{d V}{\sqrt{V^{3}}}
$$




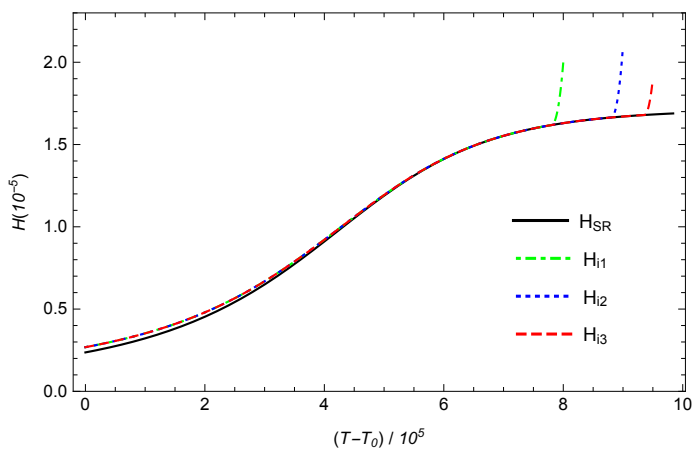

Figure 8. The solutions to the Hamilton-Jacobi equation (2.12) for the potential (3.45) with different initial values $H\left(T_{i}\right)$. The solid line labelled as $H_{\mathrm{SR}}$ corresponds to the slow-roll attractor.

Although the analytic form for $V(T)$ is not available, the potential $V(T)$ can be obtained from eq. (3.45) and it is shown in figure 3. The slow-roll attractor is shown in figure 8. If we take $\alpha=1.588, \beta=1.801$ and $N_{*}=60$, we get $n_{s}=0.968, r=0.022$ and $\Delta T=T_{*}-T_{e}=6.93 \times 10^{5}$, so the bound (2.46) is satisfied.

\section{Reheating}

From figure 3, we see that the reconstructed potentials have minimum, so when the tachyon rolls down to the minimum, inflation ends and the tachyon field begins to oscillate around the minimum. Due to the interaction between the tachyon and relativistic particles, the tachyon decays to relativistic particles and the energy stored in the tachyon field is converted to relativistic particles. Although the physics of reheating is uncertain, the reheating process may provide additional constraint on inflationary models.

The pivotal scale $k_{*}=0.002 \mathrm{Mpc}^{-1}$ is related to the current Hubble horizon as

$$
\frac{c_{s} k_{*}}{a_{0} H_{0}}=\frac{a_{*} H_{*}}{a_{0} H_{0}}=\frac{a_{*}}{a_{e}} \frac{a_{e}}{a_{r e}} \frac{a_{r e}}{a_{0}} \frac{H_{*}}{H_{0}}=e^{-N_{*}-N_{r e}} \frac{a_{r e}}{a_{0}} \frac{H_{*}}{H_{0}},
$$

where $a_{r e}$ denotes the value of the scale factor at the end of reheating, $N_{r e}$ denotes the number of $e$-folds during reheating, and we assume that radiation domination begins immediately after the reheating, and reheating begins immediately after inflation. If the equation of state parameter $w_{r e}$ is a constant during reheating, then we have

$$
N_{r e}=\frac{1}{3\left(1+w_{r e}\right)} \ln \frac{\rho_{e}}{\rho_{r e}}
$$

where $\rho_{r e}$ is related with the temperature $T_{r e}$ as

$$
\rho_{r e}=\frac{\pi^{2}}{30} g_{r e} T_{r e}^{4},
$$


and $g_{r e}$ is the effective number of relativistic species at reheating. From the entropy conservation, we can express the temperature $T_{r e}$ with the current cosmic microwave background temperature $T_{0}=2.725 \mathrm{~K}$ through the following relation

$$
a_{r e}^{3} g_{s, r e} T_{r e}^{3}=a_{0}^{3}\left(2 T_{0}^{3}+6 \times \frac{7}{8} T_{\nu 0}^{3}\right),
$$

where $g_{s, r e}$ is the effective number of relativistic species for entropy and the current neutrino temperature $T_{\nu 0}=(4 / 11)^{1 / 3} T_{0}$. Combining the above results, we get $[42,43]$

$$
\begin{gathered}
N_{r e}=\frac{4}{1-3 w_{r e}}\left[-N_{*}-\ln \frac{\rho_{e}^{1 / 4}}{H_{*}}+\frac{1}{3} \ln \frac{43}{11 g_{s, r e}}+\frac{1}{4} \ln \frac{\pi^{2} g_{r e}}{30}-\ln \frac{c_{s} k_{*}}{a_{0} T_{0}}\right], \\
T_{r e}=\exp \left[-\frac{3 N_{r e}\left(1+w_{r e}\right)}{4}\right]\left[\frac{30 \rho_{e}}{\pi^{2} g_{r e}}\right]^{1 / 4} .
\end{gathered}
$$

Since $N_{r e}$ and $T_{r e}$ depend on $g_{r e}$ and $g_{s, r e}$ logarithmically, so it is safe to take $g_{r e}=$ $g_{s, r e}=106.75$. Since at the end of inflation, $\dot{T}^{2}=2 / 3$, so $\rho_{e}=\sqrt{3} V_{e}$. Using the observational value [11]

$$
A_{s}=H_{*}^{2} /\left(8 \pi^{2} \epsilon_{*}\right)=2.2 \times 10^{-9},
$$

we get

$$
\begin{gathered}
N_{r e}=\frac{4}{1-3 w_{r e}}\left(56.94-N_{*}-\frac{1}{4} \ln V_{e}+\frac{1}{2} \ln \epsilon_{*}\right), \\
T_{r e}=\exp \left[-\frac{3 N_{r e}\left(1+w_{r e}\right)}{4}\right]\left[\frac{3 \sqrt{3} V_{e}}{10.675 \pi^{2}}\right]^{1 / 4} .
\end{gathered}
$$

These results (4.8) and (4.9) can be used to constrain inflationary models.

For the constant slow-roll inflation (3.10), at the horizon exit, we have

$$
3 H_{*}^{2}=V_{0}\left|6 e^{\eta_{V} N_{*}}-6+\eta_{V}\right|^{1 / 3} .
$$

At the end of inflation, $V_{e}=V_{0}\left|\eta_{V}\right|^{1 / 3}$, so

$$
V_{e}=24 \pi^{2} \epsilon_{*} A_{s}\left[\frac{\eta_{V} e^{-\eta_{V} N_{*}}}{6-\left(6-\eta_{V}\right) e^{-\eta_{V} N_{*}}}\right]^{1 / 3} .
$$

In deriving the above result, we used the relation $H_{*}^{2}=8 \pi^{2} \epsilon_{*} A_{s}$. Substituting eqs. (3.11) and (4.11) into eqs. (4.8) and (4.9), we get

$$
\begin{aligned}
N_{r e} & =\frac{4}{1-3 w_{r e}}\left\{60.56-\left(1-\frac{\eta_{V}}{12}\right) N_{*}+\frac{1}{6} \ln \left[\frac{\eta_{V}}{6-\left(6-\eta_{V}\right) e^{-\eta_{V} N_{*}}}\right]\right\}, \\
T_{r e} & =0.01\left[\frac{\eta_{V}}{6-\left(6-\eta_{V}\right) e^{-\eta_{V} N_{*}}}\right]^{1 / 3} \exp \left[-\frac{3 N_{r e}\left(1+w_{r e}\right)}{4}-\frac{\eta_{V} N_{*}}{12}\right] .
\end{aligned}
$$



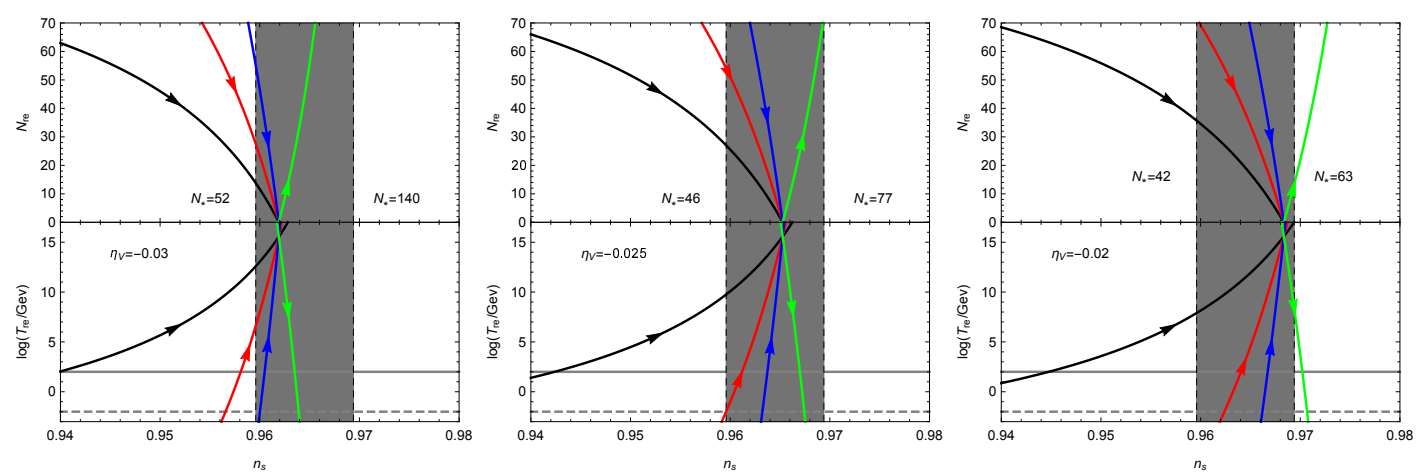

Figure 9. $N_{r e}$ (upper panels) versus $n_{s}$ as determined from (3.12) and (4.12), and $T_{r e}$ (lower panels) versus $n_{s}$ as determined from (3.12) and (4.13) for the constant slow-roll inflation. From the left to right, the parameter $\eta_{V}$ is chosen as $\eta_{V}=-0.03,-0.025$ and -0.02 , respectively. The gray band corresponds to the $1 \sigma$ Planck constraint $n_{s}=0.9645 \pm 0.0049$ [11], and the $1 \sigma$ constraint on $N_{*}$ is also given. In each panel, the black, red, blue and green lines denote $w_{r e}=-1 / 3,0,1 / 6$ and $2 / 3$, respectively, and the arrow indicates that $N_{*}$ increases along the line. The horizontal gray solid and dashed lines in lower panels correspond to the electroweak scale $T_{E W} \sim 100 \mathrm{Gev}$ and the big bang nucleosynthesis scale $T_{B B N} \sim 10$ Mev, respectively.

By choosing different values for $\eta_{V}$ and $w_{r e}$, we calculate $n_{s}, N_{r e}$ and $T_{r e}$ by varying $N_{*}$, and the results are shown in figure 9 . The parameter $\eta_{V}$ is chosen as $\eta_{V}=$ $-0.03,-0.025$ and -0.02 respectively from the left to right in figure 9 , the gray region corresponds to the $1 \sigma$ Planck constraint $n_{s}=0.9645 \pm 0.0049$ [11], and the $1 \sigma$ constraint on $N_{*}$ for the chosen value of $\eta_{V}$ is also shown. The black, red, blue and green lines denote $w_{r e}=-1 / 3,0,1 / 6$ and $2 / 3$, respectively. The horizontal gray solid and dashed lines in lower panels correspond to the electroweak scale $T_{E W} \sim 100 \mathrm{Gev}$ and the big bang nucleosynthesis scale $T_{B B N} \sim 10 \mathrm{Mev}$, respectively. From figure 9 , we see that depending on the model parameter $\eta_{V}$ and the reheating physics (the value of $w_{r e}$ ), the constraints on $N_{r e}$ and $T_{r e}$ are different. As $\eta_{V}$ becomes larger, $n_{s}$ increases, the allowed reheating epoch becomes longer for $w_{r e}=-1 / 3,0$ and $1 / 6$ while the allowed reheating epoch becomes shorter for $w_{r e}=2 / 3$. For $-0.03<\eta_{V}<-0.02$, reheating with $-1 / 3 \leq w_{r e} \leq 2 / 3$ are all consistent with the observations. Around the central value $n_{s}=0.965, \eta_{V}=-0.025$ and $N_{*}=60, w_{r e}=1 / 6$ can have a prolonged reheating epoch and $N_{r e}$ can be larger than 70 .

For the model (3.17), we consider the case $p>1$ and $p \neq 1+2 A$. Substituting eqs. (3.18) and (3.19) into eqs. (4.8) and (4.9), we get

$$
\begin{gathered}
N_{r e}=\frac{4}{1-3 w_{r e}}\left\{60.38+\frac{1}{4} \ln \left[C(p-1)^{2}+(p-1) A^{1-p}\right]-N_{*}\right. \\
\left.-\frac{p}{4} \ln \left(N_{*}+A\right)-\frac{1}{2} \ln \left[\left(N_{*}+A\right)^{1-p}+C(p-1)\right]\right\}, \\
T_{r e}=0.01\left(N_{*}+A\right)^{-p / 4}\left[\frac{p-1}{A^{1-p}+(p-1) C}\right]^{1 / 4} \exp \left[-\frac{3 N_{r e}\left(1+w_{r e}\right)}{4}\right] .
\end{gathered}
$$



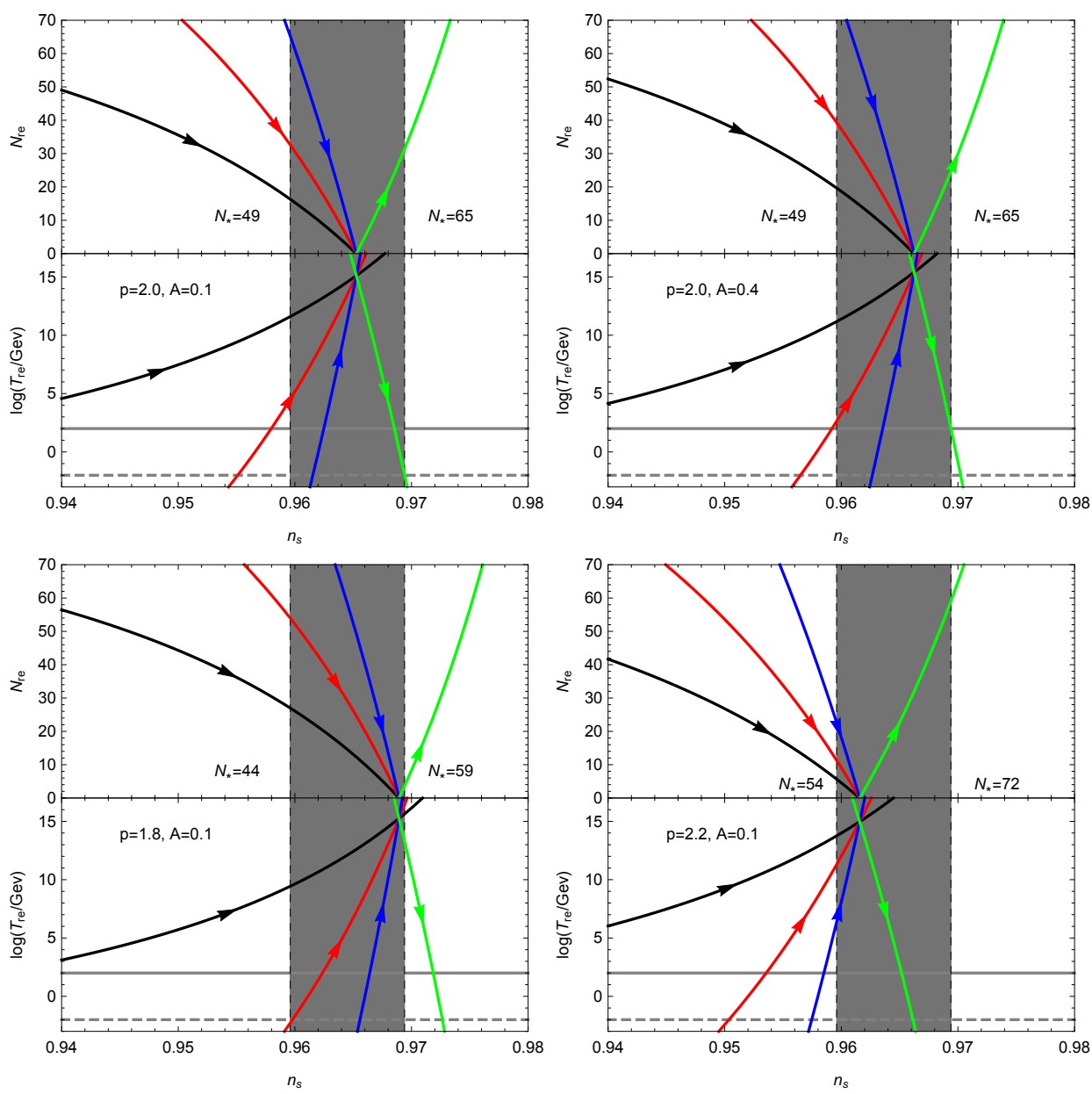

Figure 10. Same as figure 9 but for the model (3.17), the values of the model parameters $p$ an $A$ are indicated in each panel.

By choosing different values of $p, A, N_{*}$ and $w_{r e}$, we calculate $n_{s}, N_{r e}$ and $T_{r e}$ from eqs. (3.12), (4.14) and (4.15), and the results are shown in figure 10. From figure 10 , we see that depending on the model parameters $p$ and $A$ and the value of $w_{r e}$, the constraints on $N_{r e}$ and $T_{r e}$ are different, but the parameter $A$ has little impact on the reheating phase. For the parameters $p$ and $A$ that make $n_{s}$ consistent with the observation, reheating with $-1 / 3 \leq w_{r e} \leq 2 / 3$ are all consistent with the observations. As $n_{s}$ becomes larger, the allowed reheating epoch becomes longer for $w_{r e}=-1 / 3,0$ and $1 / 6$ while the allowed reheating epoch becomes shorter for $w_{r e}=2 / 3$.

For the model (3.34), we consider the case $\beta \neq 1$, Substituting eqs. (3.34) and 
(3.37) into eqs. (4.8) and (4.9), we obtain

$$
\begin{aligned}
N_{r e} & =\frac{4}{1-3 w_{r e}}\left[60.56+\frac{\alpha}{2(\beta-1)}-N_{*}-\frac{\alpha}{2(\beta-1)}\left(\frac{N_{*}+\alpha}{\alpha}\right)^{1-\beta}-\frac{\beta}{4} \ln \left(\frac{N_{*}+\alpha}{\alpha}\right)\right], \\
T_{r e} & =0.01\left(\frac{\alpha}{N_{*}+\alpha}\right)^{\beta / 4} \exp \left[-\frac{3 N_{r e}\left(1+w_{r e}\right)}{4}+\frac{\alpha}{2(1-\beta)}\left(1-\left(\frac{N_{*}+\alpha}{\alpha}\right)^{1-\beta}\right)\right] .
\end{aligned}
$$

By choosing different values of $\alpha, \beta, N_{*}$ and $w_{r e}$, we calculate $n_{s}, N_{r e}$ and $T_{r e}$ from eqs. (3.12), (4.16) and (4.17), and the results are shown in figure 11. It is obvious that depending on the model parameters $\alpha$ and $\beta$ and the value of $w_{r e}$, the constraints on $N_{r e}$ and $T_{r e}$ are different, but the parameter $\alpha$ has little impact on the reheating phase. For the parameters $\alpha$ and $\beta$ that make $n_{s}$ consistent with the observation, reheating with $-1 / 3 \leq w_{r e} \leq 2 / 3$ are all consistent with the observations. As $n_{s}$ becomes larger, the allowed reheating epoch becomes longer for $w_{r e}=-1 / 3,0$ and $1 / 6$ while the allowed reheating epoch becomes shorter for $w_{r e}=2 / 3$.

\section{Conclusions and Discussions}

Similar to the usual inflation with canonical scalar field, there is also a lower bound on the field excursion for the tachyon inflation, but the lower bound for the tachyon field depends on $A_{s}$ and $N_{*}$. Using the observational value $\ln \left(10^{10} A_{s}\right)=3.094$ [11], we derive the lower bound $\Delta T \geq 1.18 \times 10^{5}$ normalized with the reduced Planck mass $M_{p l}$ for $N_{*}=60$, and the bound are supported by the three models discussed in this work. Since the $\beta$-function $\beta(T)=-\sqrt{2 \epsilon}$, so the reconstruction of the tachyon potentials from $\beta(T)$ is equivalent to the reconstruction from the slow-roll parameter $\epsilon(T)$ or other parameterizations with the number of $e$-folds $N$. We focus on the reconstruction of tachyon potentials from the parameterizations with $N$.

Following the reconstruction procedure presented in subsection 2.4, we reconstruct three classes of tachyon potentials by parameterizing the slow-roll parameters $\epsilon$ (equivalent to the tensor to scalar ratio $r$ ), $\eta$ and the observable $n_{s}$, respectively. We first consider the case that the slow-roll parameter is a constant, we find that only the model with $\eta_{V}$ being a constant is consistent with the observations at the $1 \sigma$ level, this model is therefore called the constant slow-roll inflation. For $N_{*}=60$, we get $-0.0374<\eta_{V}<-0.0142$ at the $1 \sigma$ level, $-0.0435<\eta_{V}<-0.0031$ at the $2 \sigma$ level and $-0.0473<\eta_{V}<0.0067$ at the $3 \sigma$ level, so the concave potential is favored by the observations at more than $2 \sigma$ level. For the simple model with $n_{s}=1-p /(N+A)$, the potential is either power-law or exponential form. Since the observations constrain $A<1$, so the effect of $A$ is negligible except setting the boundary $p=1+2 A$ for the parameter $p$. The $\alpha$ attractor is the special case with $p=2$ and it is consistent with observations. For the power-law parametrization $r=16 \gamma /(N+\alpha)^{\beta}$, we find $\beta \sim 2$ is favored by the observations. For all three models, if we take $n_{s}=0.968$ and $r=0.22$, the reconstructed potentials behave similarly and they are concave potentials. 

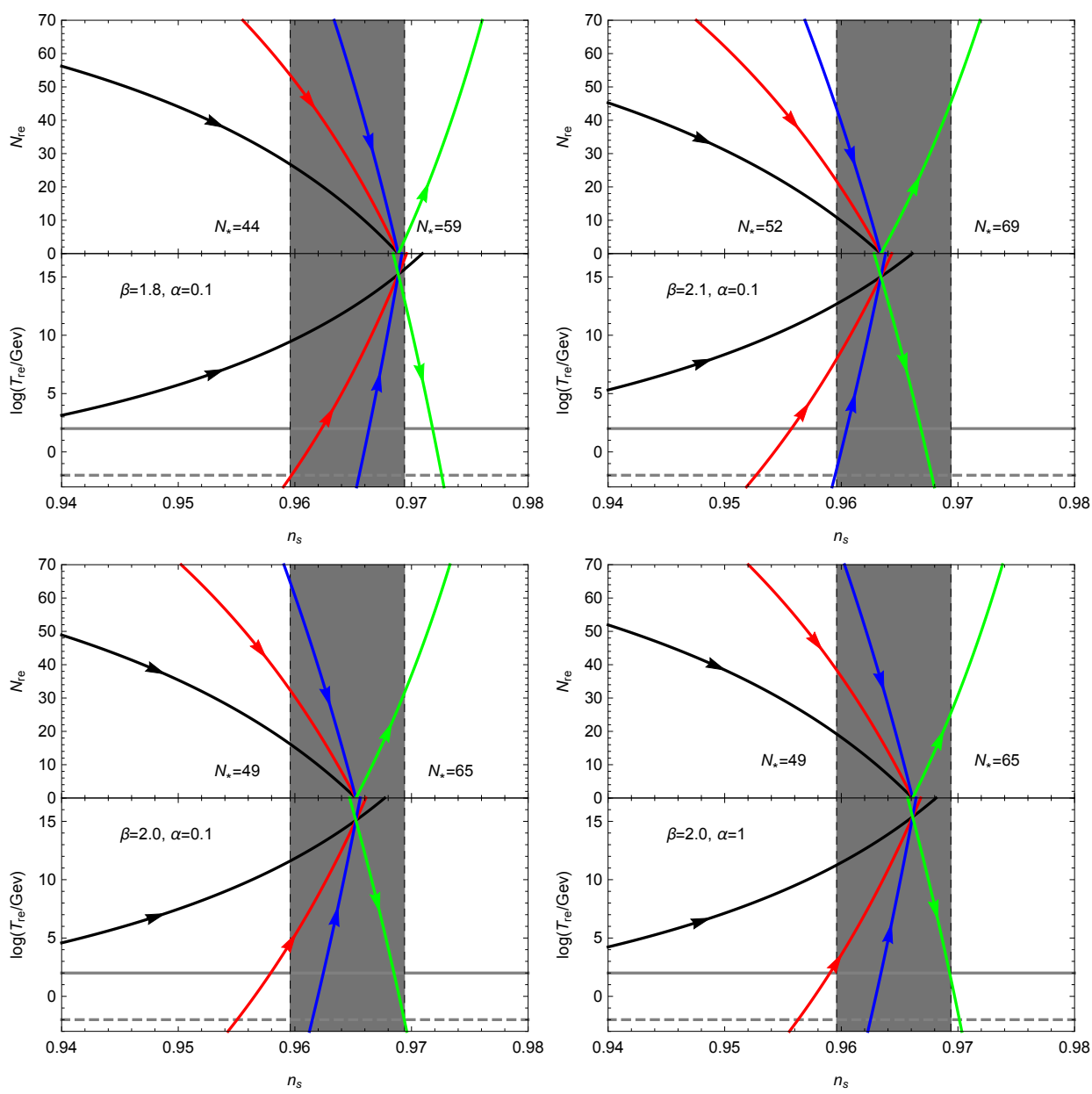

Figure 11. Same as figure 9 but for the model (3.34), the values of the model parameters $\alpha$ and $\beta$ are indicated in each panel.

Depending on the model parameters and the value of $w_{r e}$, the constraints on $N_{r e}$ and $T_{r e}$ are different, although the parameter $A$ in the model (3.17) and the parameter $\alpha$ in the model (3.34) have little impact on the reheating phase. For all three models, if we choose the model parameters so that $n_{s}$ is consistent with the observations, then reheating with $-1 / 3 \leq w_{r e} \leq 2 / 3$ are all consistent with the observations. Furthermore, as $n_{s}$ increases, the allowed reheating epoch becomes longer for $w_{r e}=-1 / 3,0$ and $1 / 6$ while the allowed reheating epoch becomes shorter for $w_{r e}=2 / 3$.

In summary, the main results are: (1) We derive the lower bound on the field excursion for the tachyon inflation, which is determined by the amplitude of the scalar perturbation $A_{s}$ and $N_{*}$. The bound is supported by all three models discussed. (2) For the models with constant slow-roll parameter, only the model with $\eta_{V}$ being a constant is consistent with the observations at the $1 \sigma$ level and concave potentials are favored by the observations. (3) As $n_{s}$ increases, the allowed reheating epoch becomes longer for $w_{r e}=-1 / 3,0$ and $1 / 6$ while the allowed reheating epoch becomes shorter for $w_{r e}=2 / 3$. 


\section{Acknowledgments}

This research was supported in part by the National Natural Science Foundation of China under Grant No. 11475065 and the Major Program of the National Natural Science Foundation of China under Grant No. 11690021.

\section{References}

[1] A. Sen, Rolling tachyon, JHEP 0204 (2002) 048, [hep-th/0203211].

[2] A. Sen, Tachyon matter, JHEP 0207 (2002) 065, [hep-th/0203265].

[3] G. W. Gibbons, Cosmological evolution of the rolling tachyon, Phys. Lett. B 537 (2002) 1-4, [hep-th/0204008].

[4] T. Padmanabhan, Accelerated expansion of the universe driven by tachyonic matter, Phys. Rev. D 66 (2002) 021301, [hep-th/0204150].

[5] A. V. Frolov, L. Kofman and A. A. Starobinsky, Prospects and problems of tachyon matter cosmology, Phys. Lett. B 545 (2002) 8-16, [hep-th/0204187].

[6] J. Garriga and V. F. Mukhanov, Perturbations in k-inflation, Phys. Lett. B 458 (1999) 219-225, [hep-th/9904176].

[7] J.-c. Hwang and H. Noh, Cosmological perturbations in a generalized gravity including tachyonic condensation, Phys. Rev. D 66 (2002) 084009, [hep-th/0206100].

[8] D. A. Steer and F. Vernizzi, Tachyon inflation: Tests and comparison with single scalar field inflation, Phys. Rev. D 70 (2004) 043527, [hep-th/0310139].

[9] A. D. Linde, Chaotic Inflation, Phys. Lett. B 129 (1983) 177-181.

[10] A. A. Starobinsky, A New Type of Isotropic Cosmological Models Without Singularity, Phys. Lett. B. 91 (1980) 99-102.

[11] Planck collaboration, P. A. R. Ade et al., Planck 2015 results. XX. Constraints on inflation, Astron. Astrophys. 594 (2016) A20, [1502.02114].

[12] Q.-G. Huang, Constraints on the spectral index for the inflation models in string landscape, Phys. Rev. D 76 (2007) 061303, [0706.2215].

[13] R. Gobbetti, E. Pajer and D. Roest, On the Three Primordial Numbers, JCAP 1509 (2015) 058, [1505.00968].

[14] V. Mukhanov, Quantum Cosmological Perturbations: Predictions and Observations, Eur. Phys. J. C 73 (2013) 2486, [1303.3925].

[15] D. Roest, Universality classes of inflation, JCAP 1401 (2014) 007, [1309.1285].

[16] J. Garcia-Bellido and D. Roest, Large- $N$ running of the spectral index of inflation, Phys. Rev. D 89 (2014) 103527, [1402.2059].

[17] J. Garcia-Bellido, D. Roest, M. Scalisi and I. Zavala, Lyth bound of inflation with a tilt, Phys. Rev. D 90 (2014) 123539, [1408.6839].

[18] J. Garcia-Bellido, D. Roest, M. Scalisi and I. Zavala, Can CMB data constrain the inflationary field range?, JCAP 1409 (2014) 006, [1405.7399]. 
[19] P. Creminelli, S. Dubovsky, D. Lpez Nacir, M. Simonovi, G. Trevisan, G. Villadoro et al., Implications of the scalar tilt for the tensor-to-scalar ratio, Phys. Rev. D 92 (2015) 123528, [1412.0678].

[20] L. Boubekeur, E. Giusarma, O. Mena and H. Ramírez, Phenomenological approaches of inflation and their equivalence, Phys. Rev. D 91 (2015) 083006, [1411.7237].

[21] L. Barranco, L. Boubekeur and O. Mena, A model-independent fit to Planck and BICEP2 data, Phys. Rev. D 90 (2014) 063007, [1405.7188].

[22] M. Galante, R. Kallosh, A. Linde and D. Roest, Unity of Cosmological Inflation Attractors, Phys. Rev. Lett. 114 (2015) 141302, [1412.3797].

[23] T. Chiba, Reconstructing the inflaton potential from the spectral index, Prog. Theor. Exp. Phys. 2015 (2015) 073E02, [1504.07692].

[24] F. Cicciarella and M. Pieroni, Universality for quintessence, JCAP 1708 (2017) 010, [1611.10074].

[25] J. Lin, Q. Gao and Y. Gong, The model independent reconstruction of inflationary potentials, Mon. Not. Roy. Astron. Soc. 459 (2016) 4029-4037, [1508.07145].

[26] S. Nojiri and S. D. Odintsov, Unified cosmic history in modified gravity: from $F(R)$ theory to Lorentz non-invariant models, Phys. Rept. 505 (2011) 59-144, [1011.0544].

[27] S. D. Odintsov and V. K. Oikonomou, Inflationary $\alpha$-attractors from $F(R)$ gravity, Phys. Rev. D94 (2016) 124026, [1612.01126].

[28] Z. Yi and Y. Gong, Nonminimal coupling and inflationary attractors, Phys. Rev. D 94 (2016) 103527, [1608.05922].

[29] S. D. Odintsov and V. K. Oikonomou, Inflation with a Smooth Constant-Roll to Constant-Roll Era Transition, 1704.02931.

[30] S. Nojiri, S. D. Odintsov and V. K. Oikonomou, Constant-roll Inflation in $F(R)$ Gravity, 1704.05945.

[31] S. Choudhury, COSMOS-e'- soft Higgsotic attractors, 1703.01750.

[32] Q. Gao and Y. Gong, Reconstruction of extended inflationary potentials for attractors, 1703.02220.

[33] R. Jinno and K. Kaneta, Hillclimbing inflation, 1703.09020.

[34] Q. Gao, The constant slow-roll inflationary model, Sci. China-Phys. Mech. Astron. 60 (2017) 090411, [1704.08559].

[35] N. C. Tsamis and R. P. Woodard, Improved estimates of cosmological perturbations, Phys. Rev. D 69 (2004) 084005, [astro-ph/0307463].

[36] W. H. Kinney, Horizon crossing and inflation with large eta, Phys. Rev. D 72 (2005) 023515, [gr-qc/0503017].

[37] J. Martin, H. Motohashi and T. Suyama, Ultra Slow-Roll Inflation and the non-Gaussianity Consistency Relation, Phys. Rev. D 87 (2013) 023514, [1211.0083].

[38] H. Motohashi, A. A. Starobinsky and J. Yokoyama, Inflation with a constant rate of roll, JCAP 1509 (2015) 018, [1411.5021]. 
[39] H. Motohashi and A. A. Starobinsky, Constant-roll inflation: confrontation with recent observational data, Europhys. Lett. 117 (2017) 39001, [1702.05847].

[40] H. Motohashi and A. A. Starobinsky, $f(R)$ constant-roll inflation, 1704.08188.

[41] N. Barbosa-Cendejas, J. De-Santiago, G. German, J. C. Hidalgo and R. R. Mora-Luna, Tachyon inflation in the Large-N formalism, JCAP 1511 (2015) 020, [1506.09172].

[42] L. Dai, M. Kamionkowski and J. Wang, Reheating constraints to inflationary models, Phys. Rev. Lett. 113 (2014) 041302, [1404.6704].

[43] J. L. Cook, E. Dimastrogiovanni, D. A. Easson and L. M. Krauss, Reheating predictions in single field inflation, JCAP 1504 (2015) 047, [1502.04673].

[44] Y. Ueno and K. Yamamoto, Constraints on $\alpha$-attractor inflation and reheating, Phys. Rev. D 93 (2016) 083524, [1602.07427].

[45] R. Kabir, A. Mukherjee and D. Lohiya, Reheating constraints on Kähler Moduli Inflation, 1609.09243.

[46] A. Di Marco, P. Cabella and N. Vittorio, Reconstruction of $\alpha$-attractor supergravity models of inflation, Phys. Rev. D 95 (2017) 023516, [1703.06472].

[47] K. Dimopoulos and C. Owen, Quintessential Inflation with $\alpha$-attractors, JCAP 1706 (2017) 027, [1703.00305].

[48] R. L. Arnowitt, S. Deser and C. W. Misner, Dynamical Structure and Definition of Energy in General Relativity, Phys. Rev. 116 (1959) 1322-1330.

[49] A. R. Liddle, P. Parsons and J. D. Barrow, Formalizing the slow roll approximation in inflation, Phys. Rev. D 50 (1994) 7222-7232, [astro-ph/9408015].

[50] D. J. Schwarz, C. A. Terrero-Escalante and A. A. Garcia, Higher order corrections to primordial spectra from cosmological inflation, Phys. Lett. B 517 (2001) 243-249, [astro-ph/0106020].

[51] D. H. Lyth, What would we learn by detecting a gravitational wave signal in the cosmic microwave background anisotropy?, Phys. Rev. Lett. 78 (1997) 1861-1863, [hep-ph/9606387].

[52] Q. Gao, Y. Gong and T. Li, Modified Lyth bound and implications of BICEP2 results, Phys. Rev. D 91 (2015) 063509, [1405.6451].

[53] P. Binetruy, E. Kiritsis, J. Mabillard, M. Pieroni and C. Rosset, Universality classes for models of inflation, JCAP 1504 (2015) 033, [1407.0820].

[54] M. Pieroni, $\beta$-function formalism for inflationary models with a non minimal coupling with gravity, JCAP 1602 (2016) 012, [1510.03691].

[55] P. Binétruy, J. Mabillard and M. Pieroni, Universality in generalized models of inflation, JCAP 1703 (2017) 060, [1611.07019].

[56] P. Brax, J. Mourad and D. A. Steer, Tachyon kinks on nonBPS D-branes, Phys. Lett. B 575 (2003) 115-125, [hep-th/0304197].

[57] L. R. W. Abramo and F. Finelli, Cosmological dynamics of the tachyon with an inverse power-law potential, Phys. Lett. B 575 (2003) 165-171, [astro-ph/0307208]. 